\title{
Tendência do vapor de água na baixa Troposfera da América do Sul
}

\author{
Water vapor trend in low Troposphere of South America
}

\author{
Henderson Silva Wanderley \\ Universidade Federal Rural do Rio de Janeiro, Brasil \\ hendynho@gmail.com
}

\section{Resumo}

O vapor de água é um constituinte importante da atmosfera por interferir na distribuição da temperatura, participar ativamente dos processos de absorção e emissão de calor sensível e latente pela atmosfera e por ser um dos principais agentes do efeito estufa. No entanto, o entendimento de como o vapor de água variou nas últimas décadas é escasso principalmente para regiões como a América do Sul. Deste modo, este estudo teve como objetivo identificar a existência de tendências na concentração de vapor de água na baixa troposfera da América do Sul. Para o desenvolvimento deste estudo foram utilizadas informações médias mensais e anuais da concentração de vapor de água troposférico, compreendidos entre os anos 1980 a 2014, analisadas para o nível bárico de 700 $\mathrm{hPa}$, provenientes de radiossondagens. As tendências foram calculadas pelo teste estatístico não paramétrico de Mann-Kendall juntamente com a análise de regressão linear mediante a utilização do teste de significância para o coeficiente angular da regressão. Os resultados mostraram dois padrões distintos de tendência para o vapor de água atmosférico. Um de aumento para as regiões Norte, Central e Leste do continente Sul Americano e um de redução para o Sul do continente.

Palavras-chave: Teste de Mann-Kendall; regressão linear; nível bárico de 700 hPa.

\begin{abstract}
Water vapor is an essential constituent of the atmosphere as it interferes with temperature distribution, actively participates in the processes of absorption and emission of sensitive and latent heat through the atmosphere, and is one of the main greenhouse effect agents. However, understanding how water vapor has varied over the last decades is scarce, especially for regions such as South America. Thus, this study objective to identify the existence of trends in water vapor concentration in the low troposphere of South America. The study was used monthly and annual average information of the concentration of tropospheric water vapor, from 1980 to 2014, analyzed for the $700 \mathrm{hPa}$ baric level, from radiosondes. The trends were calculated by the non-parametric Mann-Kendall statistical test together with the linear regression analysis using the significance test for the regression angular coefficient. The results showed two distinct trend patterns for atmospheric water vapor. One increase for the North, Central, and East regions of the South American continent and one improvement for the South of the continent.
\end{abstract}

Keywords: Mann-Kendall test; linear regression; $700 \mathrm{hPa}$ baric level.

\section{INTRODUÇÃO}

O vapor de água é um importante constituinte da atmosfera por interferir na distribuição da temperatura, participar ativamente dos processos de absorção e emissão de calor sensível pela atmosfera, atua na transferência de calor latente entre regiões e por ser um dos principais causadores do efeito estufa (CHUNG et al., 2014). Além disso, é o único componente atmosférico que muda de 
estado físico, sendo responsável pela formação e evolução das nuvens e por seus fenômenos atmosféricos importantes como a precipitação.

O vapor de água é responsável pela absorção de radiação atmosférica solar e terrestre, sendo a maior parte dessa radiação absorvida na região do infravermelho, a qual é emitida pela superfície da terrestre. O vapor de água modula os fluxos de energia no sistema climático do planeta por meio da transferência de calor latente por evaporação e condensação, modificando os fluxos de energia radiativa de ondas longas e ondas curtas do espectro eletromagnético (ALLAN, 2012).

Essa característica torna o vapor de água um componente atmosférico eficaz para o efeito estufa. Outra característica importante é que o mesmo apresentar respostas diretas ao aumento da temperatura, aumentando sua concentração na atmosfera, e por consequência sua contribuição para aquecê-la (COHEUR et al., 2003; RANDALL et al., 2007, WANDERLEY; JUSTINO, 2015).

A troca de energia entre a troposfera e a superfície realizada pelo vapor de água irá depender de sua quantidade e padrão de distribuição apresentado na atmosfera. O indicativo de diminuição ou de aumento no vapor de água atmosférico é necessário para identificar possíveis modificações no sistema climático. Entender essas respostas, em função de mudanças ocorridas no vapor de água atmosférico, pode ser essencial para identificar possíveis alterações na precipitação, saldo de radiação e temperatura (HOUGHTON et al., 2001; WULFMEYER et al., 2006).

Held e Soden (2006) apresentaram uma série de respostas climáticas que estão ligadas ao aumento da temperatura, a maior parte delas relacionada, direta ou indiretamente, a variações na concentração de vapor de água na baixa troposfera, onde estima-se que o aumento da quantidade de vapor de água contribuirá para o aquecimento global. Assim, pode-se prever com determinada margem de confiança, que algumas alterações ocorrerão na atmosfera decorrente do aumento ou redução do vapor de água na atmosfera.

A atuação do vapor de água na atmosfera vem sendo largamente discutidas, sobretudo o que diz respeito da atuação do vapor de água como feedback positivo, ou seja, sua capacidade de amplificar a ação dos gases de efeito estufa. A ação do vapor de água como feedback positivo foi ratificada por estudos como o de Dessler et al. (2009), os quais encontraram mudanças no balanço de radiação decorrente da presença do vapor de água de $2,04 \mathrm{Wm}^{-2} \mathrm{~K}^{-1}$, entre os anos de 2003 a 2008. Este estudo mostra concordância como os de Forster e Collins (2004), Minschwaner e Dessler (2004) e Minschwaner et al. (2006), que também ratificam a desempenho do vapor de água atmosférico como feedback positivo.

Segundo o relatório apresentado pelo IPCC (2013) a forçante radiativa obtida pelo aumento da concentração do dióxido de carbono $\left(\mathrm{CO}_{2}\right)$, entre os anos de 1750 a 2006, foi igual a $1,6 \mathrm{Wm}^{-2}$, valor inferior ao resultado apresentado por Dessler et al. (2009). Este resultado 
demonstra a importância do vapor de água na atmosfera e ressalta sua atuação no sistema climático. Deste modo, o entendimento do vapor de água no balanço de radiação atmosférica é essencial e pode contribuir para determinar e modificar o clima do planeta (SITCH et al., 2007; MERCADO et al., 2009).

A concentração de vapor de água na alta troposfera e baixa estratosfera apresentou um aumento gradual nas latitudes tropicais de mais de 1 ppm entre os anos de 1980-2000 (SOLOMON et al., 2010). Shindell (2001) e Forster e Collins (2004) sugerem que as mudanças no vapor de água na alta troposfera e baixa estratosfera colaboraram para as alterações climáticas observadas neste período, contribuiu para aumentar o aquecimento observado durante 1980 a 2000.

No entanto, a partir do ano 2000 foi documentada uma diminuição de 0,4 ppm da concentração de vapor de água na alta troposfera e baixa estratosfera. Essa redução no vapor de água na alta troposfera e baixa estratosfera fez contribuições substanciais para a diminuição da tendência de aquecimento global desde 2000 (SOLOMON et al., 2010).

A redução do vapor de água na estratosfera mantém-se relativamente constante a partir de 2001 até o final de 2007, embora apresente alguns indícios de um ligeiro aumento a partir de meados de 2008 (SOLOMON et al., 2010). Entretanto, algumas respostas sobre a tendência do vapor de água na baixa e média atmosfera permanecem escassas, surgindo assim, a necessidade de estudos mais específicos que visem quantificar essa variabilidade e seus impactos sobre o sistema climático. Deste modo, este estudo teve como objetivo identificar a existência de tendências na concentração de vapor de água na baixa troposfera da América do Sul.

\section{METODOLOGIA}

Para o desenvolvimento deste estudo foram utilizadas informações médias mensais e anuais da concentração de vapor de água troposférico, analisadas para o nível bárico de $700 \mathrm{hPa}$, provenientes de radiossondagens. As medições foram realizadas diariamente em 14 estações distribuídas espacialmente na América do Sul (Figura 1), onde o vapor de água troposférico foi analisado por meio da razão de mistura (r), com dados compreendidos entre os anos 1980 a 2014. Os dados utilizados foram adquiridos junto ao banco de dados de Universidade de Wyoming (http://weather.uwyo.edu/upperair/sounding.html). 


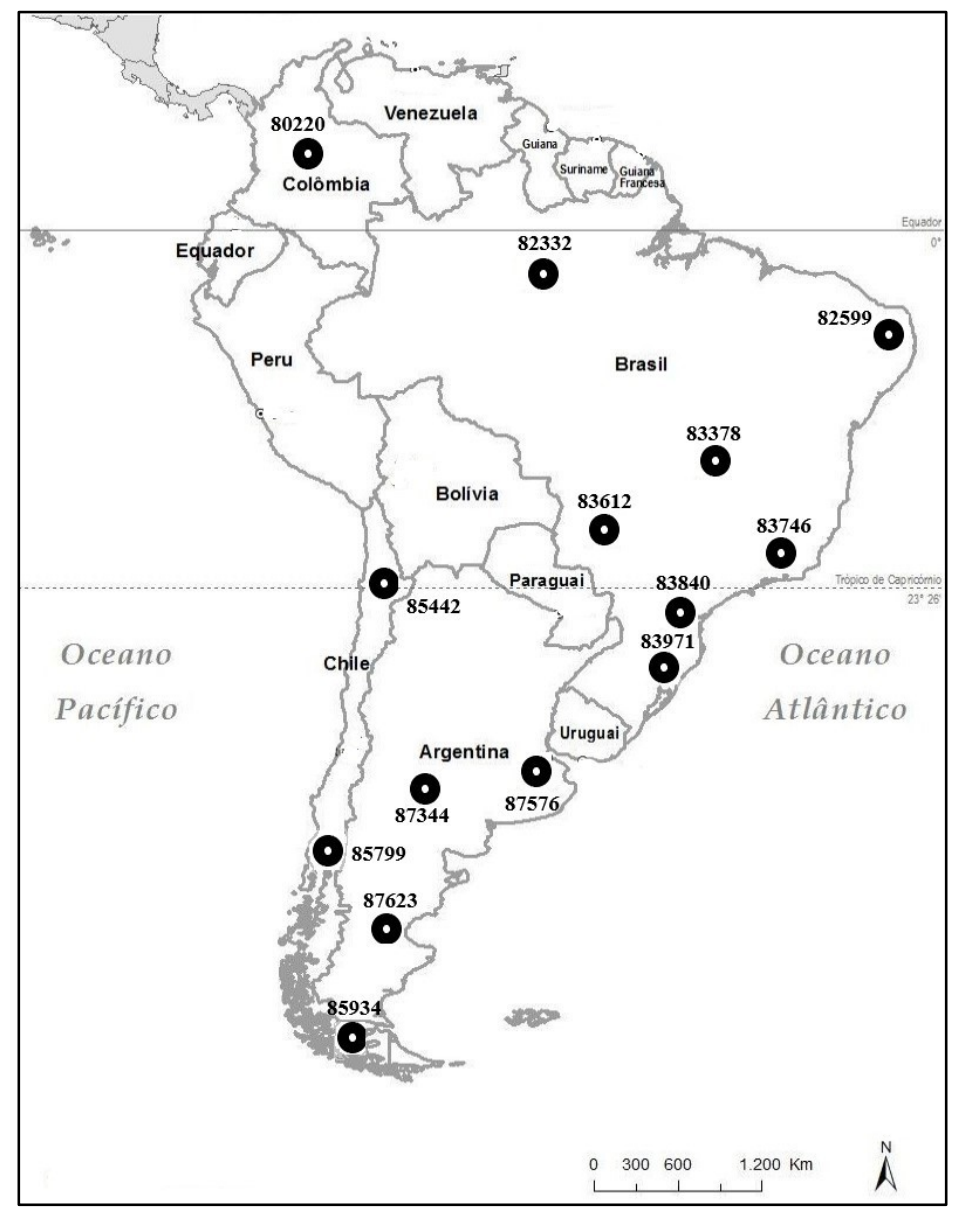

Figura 1 - Distribuição espacial e identificação das estações de medições utilizadas no estudo.

Para a identificação de tendência, as séries temporais de vapor de água mensais e anuais foram submetidas ao teste estatístico não paramétrico de Mann-Kendall (MANN, 1945; KENDALL, 1975), também conhecido como teste "S". O teste considera a hipótese de estabilidade de uma série temporal, onde a sucessão de valores ocorre de forma independente e a distribuição de probabilidade deve permanecer sempre a mesma, ou seja, uma série aleatória simples. O teste considerando a série temporal Yi de $\mathrm{n}$ termos $(1 \leq \mathrm{i} \leq \mathrm{n})$, onde a estatística desse teste é dada pela Equação (1).

$$
S=\sum_{i=1}^{n-1} \sum_{j=i+1}^{n} \operatorname{sinal}\left(Y_{j}-Y_{i}\right)
$$

em que:

$$
\operatorname{sinal}\left(Y_{j}-Y_{i}\right)=\left\{\begin{array}{ll}
+1 & \operatorname{se}\left(Y_{j}-Y_{i}\right)>0 \\
0 & \operatorname{se}\left(Y_{j}-Y_{i}\right)=0 \\
-1 & \operatorname{se}\left(Y_{j}-Y_{i}\right)<0
\end{array}\right\}
$$


Para séries com grande número de termos (n), a estatística "S" apresenta uma distribuição normal com média zero e variância definida pela Equação (2).

$$
\operatorname{Var}(\mathrm{S})=\frac{\mathrm{n}(\mathrm{n}-1)(2 \mathrm{n}+5)}{18}
$$

O teste tem como hipótese nula $\left(\mathrm{H}_{\mathrm{o}}\right)$ a ausência de tendência na série temporal em análise. Testando a significância estatística de $\mathrm{S}$ para a hipótese alternativa $\left(\mathrm{H}_{1}\right)$, a qual expressa à existência de tendência, usa-se um teste bilateral a $\mathrm{H}_{1}$, pelo qual a hipótese alternativa pode ser rejeitada por meio da estatística Z, Equação (3).

$$
z= \begin{cases}\frac{(S-1)}{\sqrt{\operatorname{Var}(S)}} & \text { se } S>0 \\ 0 & \text { se } S=0 \\ \frac{(S+1)}{\sqrt{\operatorname{Var}(S)}} & \text { se } S<0\end{cases}
$$

A decisão de aceitar a hipótese nula $\left(\mathrm{H}_{\mathrm{o}}\right)$ de ausência de tendência, e assim confirmar a hipótese de estabilidade dos dados é obtida por meio da utilização da análise da estatística Z. O teste tem como hipótese alternativa $\left(\mathrm{H}_{1}\right)$, a existência de tendência na série temporal em questão.

$O$ sinal da estatística $Z$ indica se a tendência é crescente $(Z>0)$ ou decrescente $(Z<0)$. Em um teste bilateral para tendência, $H_{1}$ deve ser aceita se $|Z| \geq Z_{\alpha / 2}$, em que o valor de $Z_{\alpha / 2}$ é obtido da tabela na distribuição normal padronizada. Para esse teste foi adotado nível de significância de $\alpha_{o}=$ $0,05 \%$ de probabilidade.

A análise de regressão também foi realizada para indicar alterações na série temporal mediante a utilização do teste de significância para o coeficiente angular da reta. Esse teste avalia se esse coeficiente é significantemente diferente de zero, indicando a presença de tendência linear, sendo calculada pela regressão linear de Y com variável aleatória no tempo X, Equação (4).

$$
\mathrm{Y}=\mathrm{a}+\mathrm{bX}
$$

sendo:

Y - variável em análise

$\mathrm{X}$ - tempo

a e b - coeficientes da regressão calculados pelo método dos mínimos quadrados 
A hipótese nula $\left(\mathrm{H}_{\mathrm{o}}\right)$ de que não existe tendência, ou seja, $\mathrm{b}=0$ foi testada por meio do teste $\mathrm{t}$ de Student com n-2 graus de liberdade, Equação (5). A hipótese de nulidade é rejeitada quando o valor de $\mathrm{t}$ calculado é maior, em valor absoluto, ao valor crítico tabelado $t_{\alpha / 2}, \mathrm{n}-2$, a um determinado nível de significância $\alpha_{0}$, sendo para esse teste também adotado nível de significância de $\alpha_{\mathrm{o}}=0,05 \%$.

$$
\mathrm{t}=\frac{\mathrm{r} \sqrt{\mathrm{n}-2}}{\sqrt{1-\mathrm{r}^{2}}}=\frac{b}{s / \sqrt{S S x}}
$$

em que:

$\mathrm{n}$ - tamanho da amostra

r - coeficiente de correlação de Pearson

s - desvio padrão dos resíduos

$\mathrm{b}$ - coeficiente angular da reta

SSX - soma dos quadrados da variável independente (tempo em análise de tendência)

\section{RESULTADOS E DISCUSSÕES}

Na América do Sul foi identificado dois padrões distintos de tendência na média anual da concentração de vapor de água em $700 \mathrm{hPa}$. O primeiro padrão, de aumento no vapor de água, foi identificado na tendência da média anual da concentração de vapor de água na atmosfera para as estações localizadas nas regiões Norte, Nordeste, Central e Leste do continente Sul Americano, com quase a totalidade das estações inseridas no Brasil, incluindo a floresta Amazônica, demonstraram esse padrão (Tabela 1).

Nas três estações localizadas ao Norte da América do Sul foi evidenciada tendência estaticamente significativa na média anual de vapor de água em duas delas, as estações 82332, 82599, juntamente com a estação 83840 localizada ao Leste da América. Esse acréscimo foi de 0,22 $\mathrm{gkg}^{-1}$ por década na estação 82332, sendo este o maior aumento anual observado para as estações analisadas. Para as estações 82599 e 83840 o aumento decadal na média anual no vapor de água foi de $0,17 \mathrm{gkg}^{-1}$.

O padrão observado nas estações 80222,82332 e 82599 foi semelhante, com vários meses apresentando significância estatística. Parte do aumento no vapor de água atmosférico nestes meses ser explicada pela variabilidade natural do sistema climático (BARKHORDARIAN et al., 2019). Esse aumento pode ser resultado também da convecção profunda, a qual é o principal contribuinte para mudanças na precipitação tropical (TAN et al., 2015). Projeções futuras mostram que a 
precipitação líquida média aumentará significativamente na faixa tropical (LEVANG; SCHMITT, 2015).

Tabela 1 - Teste estatístico de Mann-Kendall (Z), teste de Student (t), coeficiente angular da reta $(\beta)$, aplicados à concentração de vapor de água no nível de $700 \mathrm{hPa}$ para as estações localizadas na América do Sul.

\begin{tabular}{|c|c|c|c|c|c|c|c|c|c|c|c|c|c|}
\hline & Jan & Fev & Mar & Abr & Mai & Jun & Jul & Ago & Set & Out & Nov & Dez & Ano \\
\hline \multicolumn{14}{|c|}{ Estação 80222 - Bogotá, Colômbia } \\
\hline $\mathrm{Z}^{3}$ & $-0,13$ & $-0,37$ & $2,53 *$ & $-0,93$ & 0,15 & 0,32 & 0,08 & 0,68 & 0,44 & 1,27 & 0,64 & 0,84 & 0,98 \\
\hline $\mathrm{t}$ & $-0,17$ & 0,00 & $2,69^{*}$ & $-1,16$ & 0,66 & 0,43 & $-0,08$ & 0,82 & 0,36 & 0,99 & 0,86 & 0,84 & 1,13 \\
\hline$\beta_{10}$ & $-0,03$ & 0,00 & 0,46 & $-0,22$ & 0,15 & 0,07 & $-0,01$ & 0,09 & 0,04 & 0,15 & 0,14 & 0,13 & 0,08 \\
\hline \multicolumn{14}{|c|}{ Estação 82332 - Manaus, Brasil } \\
\hline Z & $-0,76$ & 0,30 & 0,93 & $2,20^{*}$ & 1,30 & $2,97 *$ & $2,70^{*}$ & 1,88 & 1,95 & 1,68 & 1,27 & $2,00^{*}$ & $3,15^{*}$ \\
\hline $\mathrm{t}$ & $-0,55$ & $-0,33$ & 0,80 & $2,25^{*}$ & 1,07 & $2,96^{*}$ & $2,87^{*}$ & $2,24 *$ & $2,50 *$ & $2,13^{*}$ & 1,45 & 2,04 & $4,11^{*}$ \\
\hline$\beta_{10}$ & $-0,05$ & $-0,05$ & 0,08 & 0,28 & 0,14 & 0,63 & 0,44 & 0,31 & 0,26 & 0,24 & 0,17 & 0,26 & 0,22 \\
\hline \multicolumn{14}{|c|}{ Estação 82599 - Natal, Brasil } \\
\hline $\mathrm{Z}$ & 1,92 & 0,45 & 0,44 & $2,95 *$ & 0,86 & $2,41 *$ & 0,44 & $-0,11$ & $-0,30$ & 1,13 & $2,34 *$ & 0,78 & $2,37 *$ \\
\hline $\mathrm{t}$ & 1,99 & 0,45 & $-0,05$ & $3,42 *$ & 1,32 & 1,92 & $-0,52$ & 0,04 & 0,03 & 1,12 & $2,44 *$ & 1,05 & $2,38 *$ \\
\hline$\beta_{10}$ & 0,36 & 0,08 & 0,00 & 0,59 & 0,26 & 0,36 & $-0,12$ & 0,00 & 0,00 & 0,13 & 0,22 & 0,13 & 0,17 \\
\hline \multicolumn{14}{|c|}{ Estação 83378 - Brasília, Brasil } \\
\hline$Z^{3}$ & 0,52 & 1,00 & 0,42 & 0,66 & $-0,25$ & $-1,02$ & 0,39 & $-1,47$ & $-0,56$ & 0,42 & 1,46 & 0,20 & 0,06 \\
\hline $\mathrm{t}$ & 0,62 & 1,92 & $-0,02$ & 1,03 & $-0,65$ & $-0,43$ & 0,10 & $-1,43$ & $-0,73$ & 0,44 & 1,76 & $-0,26$ & 0,43 \\
\hline$\beta_{10}$ & 0,08 & 0,26 & 0,00 & 0,18 & $-0,08$ & $-0,07$ & 0,02 & $-0,34$ & $-0,14$ & 0,05 & 0,19 & $-0,02$ & 0,03 \\
\hline \multicolumn{14}{|c|}{ Estação 83612 - Campo Grande, Brasil } \\
\hline$Z^{\prime}$ & $-1,00$ & $-0,54$ & $-1,64$ & $-2,34 *$ & $-0,74$ & $-1,68$ & 1,15 & $-2,41 *$ & $-0,17$ & 0,59 & $2,36^{*}$ & 0,47 & $-0,95$ \\
\hline $\mathrm{t}$ & $-1,47$ & $-0,91$ & $-1,57$ & $-2,36^{*}$ & $-0,91$ & $-1,70$ & 1,52 & $-2,02$ & $-0,30$ & 0,50 & $2,55^{*}$ & 0,79 & $-1,41$ \\
\hline$\beta_{10}$ & $-0,23$ & $-0,12$ & $-0,23$ & $-0,23$ & $-0,16$ & $-0,26$ & 0,29 & $-0,39$ & $-0,04$ & 0,08 & 0,36 & 0,12 & $-0,07$ \\
\hline \multicolumn{14}{|c|}{ Estação 83746 - Rio de Janeiro, Brasil } \\
\hline Z & $2,19 *$ & $2,20^{*}$ & 1,97 & 0,28 & $-0,56$ & $-0,69$ & 0,81 & $-1,37$ & 0,40 & 1,13 & $2,38^{*}$ & $-0,11$ & 1,59 \\
\hline $\mathrm{t}$ & $2,43 *$ & $2,43^{*}$ & 1,82 & 0,72 & $-0,68$ & $-1,03$ & 1,11 & $-1,00$ & $-0,36$ & 1,84 & $2,33^{*}$ & $-0,17$ & 1,69 \\
\hline$\beta_{10}$ & 0,31 & 0,41 & 0,29 & 0,10 & $-0,10$ & $-0,12$ & 0,14 & $-0,14$ & $-0,08$ & 0,30 & 0,26 & $-0,03$ & 0,11 \\
\hline \multicolumn{14}{|c|}{ Estação 83840 - Curitiba, Brasil } \\
\hline $\mathrm{Z}$ & $2,75^{*}$ & 0,84 & 1,34 & 1,90 & $-0,73$ & 0,78 & $2,00 *$ & $-0,48$ & 1,81 & 1,05 & 0,44 & 0,00 & $2,95^{*}$ \\
\hline $\mathrm{t}$ & $3,23 *$ & 1,38 & 1,55 & 1,74 & $-0,83$ & 0,40 & 1,68 & $-0,61$ & 1,50 & 1,87 & 1,80 & 0,04 & $3,66^{*}$ \\
\hline$\beta_{10}$ & 0,54 & 0,21 & 0,21 & 0,30 & $-0,11$ & 0,04 & 0,21 & $-0,10$ & 0,18 & 0,39 & 0,19 & 0,00 & 0,17 \\
\hline \multicolumn{14}{|c|}{ Estação 83971 - Porto Alegre, Brasil } \\
\hline $\mathrm{Z}$ & 0,88 & 0,40 & 1,46 & 0,00 & $-1,41$ & 1,18 & $-0,10$ & 0,00 & 0,95 & 0,00 & $-0,68$ & 0,18 & 0,54 \\
\hline $\mathrm{t}$ & 1,05 & 0,22 & 1,61 & 0,00 & $-1,64$ & 0,78 & $-0,28$ & 0,17 & 0,71 & 0,30 & 0,16 & $-0,19$ & 0,75 \\
\hline$\beta_{10}$ & 0,16 & 0,04 & 0,25 & 0,00 & $-0,23$ & 0,08 & $-0,03$ & 0,01 & 0,08 & 0,04 & 0,01 & $-0,01$ & 0,03 \\
\hline \multicolumn{14}{|c|}{ Estação 85442 - Antofagasta, Chile } \\
\hline$Z^{3}$ & 0,33 & $-0,16$ & $-0,54$ & 0,52 & $-1,00$ & $-1,32$ & $-1,31$ & $-1,44$ & $-2,65^{*}$ & $-2,15^{*}$ & $-2,36^{*}$ & $-0,44$ & $-1,24$ \\
\hline $\mathrm{t}$ & 0,34 & $-0,39$ & $-0,38$ & 0,82 & $-0,91$ & $-1,32$ & $-0,98$ & $-1,54$ & $-3,16^{*}$ & $-2,42 *$ & $-2,44 *$ & $-0,98$ & $-1,71$ \\
\hline$\beta_{10}$ & 0,06 & $-0,07$ & $-0,07$ & 0,06 & $-0,06$ & $-0,08$ & $-0,07$ & $-0,10$ & $-0,18$ & $-0,17$ & $-0,18$ & $-0,09$ & $-0,08$ \\
\hline \multicolumn{14}{|c|}{ Estação 85799 - Puerto montt Chile } \\
\hline $\mathrm{Z}$ & 0,32 & 0,00 & 1,00 & $-1,73$ & $-0,27$ & $-0,05$ & $-0,81$ & 0,35 & $-1,73$ & 0,06 & 0,71 & $-0,40$ & $-0,54$ \\
\hline $\mathrm{t}$ & 0,17 & 0,18 & 1,08 & $-1,82$ & $-0,27$ & 0,14 & $-0,69$ & 0,24 & $-1,61$ & 0,06 & 0,80 & $-0,17$ & $-0,30$ \\
\hline$\beta_{10}$ & 0,00 & 0,01 & 0,10 & $-0,12$ & $-0,02$ & 0,01 & $-0,04$ & 0,01 & $-0,08$ & 0,00 & 0,05 & $-0,01$ & 0,00 \\
\hline \multicolumn{14}{|c|}{ Estação 85934 - Punta Arena, Chile } \\
\hline$Z^{\prime}$ & $-0,39$ & $-0,71$ & 0,32 & 0,52 & $-1,34$ & 1,25 & $-1,07$ & $2,12^{*}$ & 0,54 & $-1,98^{*}$ & $-1,90$ & $-2,04 *$ & $-1,20$ \\
\hline $\mathrm{t}$ & $-0,92$ & $-0,78$ & 0,24 & 0,68 & $-0,98$ & 0,75 & $-1,29$ & $2,16^{*}$ & 0,58 & $-1,97$ & $-1,71$ & $-1,63$ & $-1,25$ \\
\hline$\beta_{10}$ & $-0,07$ & $-0,04$ & 0,01 & 0,03 & $-0,04$ & 0,03 & $-0,05$ & 0,06 & 0,02 & $-0,07$ & $-0,07$ & $-0,08$ & $-0,02$ \\
\hline \multicolumn{14}{|c|}{ Estação 87344 - Cordoba, Argentina } \\
\hline$Z^{3}$ & $-1,41$ & 0,83 & 0,66 & 0,64 & $-0,95$ & $-1,88$ & $-4,08^{*}$ & $-2,68 *$ & $-0,66$ & $-1,13$ & 0,00 & 0,27 & $-1,27$ \\
\hline $\mathrm{t}$ & $-1,88$ & 0,18 & 0,86 & 0,45 & $-1,01$ & $-2,19 *$ & $-5,39 *$ & $-3,36^{*}$ & $-0,71$ & $-1,26$ & $-0,11$ & 0,41 & $-2,04$ \\
\hline$\beta_{10}$ & $-0,22$ & 0,02 & 0,14 & 0,05 & $-0,08$ & $-0,17$ & $-0,40$ & $-0,32$ & $-0,09$ & $-0,13$ & $-0,01$ & 0,56 & $-0,09$ \\
\hline \multicolumn{14}{|c|}{ Estação 87576 - Buenos Aires, Argentina } \\
\hline $\mathrm{Z}$ & $-1,66$ & 0,06 & $-1,63$ & $-2,70 *$ & $-2,15^{*}$ & $-1,22$ & $-1,17$ & $-1,51$ & $-0,64$ & $-2,10^{*}$ & $-1,93$ & $-3,29 *$ & $-3,90 *$ \\
\hline $\mathrm{t}$ & $-1,68$ & $-0,25$ & $-1,49$ & $-3,68 *$ & $-2,14 *$ & $-1,20$ & $-1,18$ & $-1,55$ & $-0,82$ & $-2,22 *$ & $-1,89$ & $-3,74 *$ & $-4,75^{*}$ \\
\hline$\beta_{10}$ & $-0,15$ & $-0,03$ & $-0,21$ & $-0,37$ & $-0,24$ & $-0,11$ & $-0,09$ & $-0,13$ & $-0,08$ & $-0,24$ & $-0,19$ & $-0,35$ & $-0,18$ \\
\hline \multicolumn{14}{|c|}{ Estação 87623 - Santa Rosa, Argentina } \\
\hline$Z^{3}$ & 0,10 & 1,27 & $-0,44$ & $-1,93$ & $-0,57$ & $-2,43^{*}$ & $-1,61$ & $-1,66$ & $-0,74$ & 0,00 & 0,81 & $-0,13$ & $-1,15$ \\
\hline $\mathrm{t}$ & $-0,46$ & 1,39 & $-0,41$ & $-2,69 *$ & $-0,44$ & $-1,06$ & $-1,61$ & $-1,21$ & $-1,21$ & 0,22 & 0,72 & 0,11 & $-1,15$ \\
\hline$\beta_{10}$ & $-0,03$ & 0,17 & $-0,05$ & $-0,28$ & $-0,04$ & $-0,14$ & $-0,13$ & $-0,07$ & $-0,09$ & 0,02 & 0,05 & 0,01 & $-0,05$ \\
\hline
\end{tabular}


Mudanças observados sobre a floresta é fundamental para examinar as mudanças no sistema climático devido ao seu papel significativo nos ciclos globais de carbono e água, além de fortes mecanismos de retroalimentação que podem potencialmente exacerbar as mudanças climáticas. As mudanças que ocorrem na floresta dependem fortemente da demanda de água na atmosfera. O aumento previsto da demanda atmosférica de água devido ao aquecimento global fará com que as secas na Amazônia se tornem mais generalizadas e severas, com estações secas sobre as América do Sul tropicais mais quentes (BARKHORDARIAN et al., 2017).

Nas estações que demonstraram tendência positiva na média anual de vapor de água, a variação mensal da concentração foi de aumento para quase todos os meses, sendo em vários casos, observada tendência estaticamente significativa para esse aumento. Na estação 82332, apenas o mês de janeiro apresentou tendência negativa, no entanto essa sem significância estatística. Para os demais meses a tendência foi positiva e significativa para os meses de abril, junho a outubro e dezembro, para pelo menos um dos testes estatísticos realizados.

Esse padrão observado na estação 82332 de poucos meses demostrando tendência negativa foi observado em todas as estações que apresentaram tendência positiva na média anual. Para essas estações tendências estatisticamente significativas para vários meses também foram observadas. Tendência de aumento no vapor de água tropical também foi documentada por Paltridge et al. (2009) e Barkhordarian et al. (2019).

O incremento mensal de vapor de água na troposfera da América do Sul geralmente está inserido nos períodos onde se observa os maiores índices pluviométricos na região em análise. Essas chuvas são acompanhadas por movimentos de ar ascendente e ventos fracos, caraterísticos dos processos convectivos, típicos das regiões tropicais, com intenso transporte de energia e massa para a atmosfera (FISCH et al., 2007; CORRÊA et al., 2008).

Além destes processos, destacam-se sistemas de escala sinótica, que contribuem com o transporte de vapor de água nas regiões tropicais como: a Zona de Convergência Intertropical (ZCIT), Zona de Convergência do Atlântico Sul (ZCAS), Alta da Bolívia (AB), dentre outros. Consoante a estes sistemas, existe mudança no padrão de circulação atmosférica, que envolve variação na circulação de Walker e Hadley, em função de anomalias de temperatura da superfície dos oceanos equatoriais, como os que causaram secas e enchentes na Amazônia (MARENGO et al., 2008; ANDREOLI et al., 2012, ENGLAND et al., 2014).

A região central do Brasil é caracterizada por apresentar convergência de fluxo de vapor de água junto ao continente no setor ocidental do Atlântico Sul, onde a circulação é dominada pelos anticiclones do Pacífico e Atlântico Sul, principalmente durante o verão, como demonstrado nas tendências obtidas para essas estações. Esse fluxo produz convergência no vapor de água 
consistente com a máxima precipitação que ocorre nesta região, caraterizadas pela Zona de Convergência do Atlântico Sul (ZCAS), (LABRAGA et al., 2000). Esse aumento de vapor de água pode ser observado para alguns meses com tendência estatisticamente significativa.

Destaca-se também a influência da alta subtropical do Atlântico Sul, no transporte de umidade da região oeste do oceano Atlântico tropical para a bacia Amazônica. A esse vapor de água soma-se o que é evapotranspirado pela floresta tropical. O transporte de umidade atmosférico continua sendo observado até na região extratropical da América do Sul, mais especificamente na Bacia do Rio da Prata, distante mais de 3.000 km (VERA et al., 2006).

Esse transporte de umidade contribui para as tendências positivas de vapor de água observadas sobre quase todo o Brasil. No final do verão, no Hemisfério Sul, é observado uma diminuição da umidade provinda da Amazônia e oceano Atlântico ratificando as tendências negativas na região central do Brasil neste período (VEIGA et al., 2002).

A convecção na região tropical é o principal mecanismo para exportar o excesso de energia para as regiões de latitude maiores. Os fluxos decorrentes da convecção geram movimentos ascendentes e formam nuvens convectivas. As nuvens constituem um dos principais componentes do sistema climático para a determinação da quantidade de energia solar absorvida pela superfície terrestre, da radiação térmica emitida para o espaço e dos processos de feedback do sistema climático, além de amplificar a atuação do vapor de água na atmosfera.

O acréscimo observado no vapor de água tem o potencial de causar aumento na temperatura da parte mais baixa da atmosfera. Esse aquecimento é resultando do efeito de feedback do vapor de água na troposfera. Outra questão relevante ao aumento da temperatura atmosférica se dá à atuação das nuvens. As nuvens atuam intensificando o feedback do vapor de água na atmosfera, aumentando o caminho óptico do espectro solar na atmosfera por meio da reflexão (SODEN et al., 2008). As projeções realizadas por Minschwaner e Dessler (2004), para os próximos 100 anos, mostraram que o feedback do vapor de água será responsável por um aumento de $5,8^{\circ} \mathrm{C}$ na temperatura do planeta, em relação ao aumento total de $7^{\circ} \mathrm{C}$, simulado pelo autor.

Chung et al. (2014) mostraram que o aumento observado no vapor de água na troposfera nas últimas três décadas foi atribuído principalmente às atividades humanas. Essa atribuição tem implicações significativas para as ciências climáticas, pois corrobora a presença do maior feedback positivo no sistema climático.

O outro padrão observado, o de redução na média anual do vapor de água, abrange todo Sul da América do Sul, compreendida pelo Chile a Argentina. A única exceção está na estação 83612, localizada no Brasil, que também demonstrou redução no vapor de água anual e nos meses de abril e agosto com tendência significativa para esse decréscimo. 
As estações localizadas no Sul do continente Sul Americano, embora mostrem redução na concentração de vapor de água, apenas na estação 87576, esse decréscimo foi estatisticamente significativo. No entanto, a redução mensal de vapor de água foi ratificada em várias estações, com algumas mostrando tendência negativa em quase todos os meses, com tendência estaticamente significativa sendo observada.

A maior redução na concentração anual de vapor de água foi observada na estação 87576 , com um decréscimo de $0,18 \mathrm{gkg}^{-1}$ por década. Nesta estação, o mês de abril apresentou a maior redução mensal no vapor de água, sendo está de $0,37 \mathrm{gkg}^{-1}$ por década. Na estação 87344 , também localizada na Argentina, foi observada a maior redução mensal entre todas as estações analisadas neste estudo. Esse decréscimo foi indicado para o mês de julho, com um decréscimo de $0,40 \mathrm{gkg}^{-1}$ por década.

O decréscimo observado no vapor de água no Sul do continente americano foi maior do que o aumento observado na região Norte deste continente. Tendo em vista que a quantidade de vapor de água é menor em latitudes maiores, esse resultado aponta uma maior modificação do vapor de água nesta região.

A ocorrência de meses com tendência significância negativa na concentração de vapor de água está compreendida para o período de abril a dezembro, embora em alguns casos, seja observada tendência negativa em meses diferentes, como janeiro. Entretanto, esse comportamento não pode ser caracterizado como um padrão, pois em quase todos os meses foi observado tendência de redução na concentração de vapor de água mensal.

Esses resultados estão coerentes com as apresentados por Cai et al. (2012) os quais mostraram que desde o final da década de 1970, regiões semiáridas do Hemisfério Sul, como Chile e Argentina, África do Sul e Austrália, têm experimentado uma tendência de secagem nos meses de outono, predominantemente durante abril e maio. A redução das chuvas neste período coincide com os meses de expansão em direção aos polos da célula de Hadley. Todavia, somente para o setor Sudeste da Austrália foi confirmada essa influência, com redução da precipitação.

Para a África do Sul o estudo de Cai et al. (2012) comprovam que a região não é diretamente afetada pela variabilidade da borda da célula de Hadley em qualquer período, embora seja observada uma mudança no padrão da precipitação em direção ao polo. Para o Chile, não se pode atribuir a redução da precipitação a uma expansão nas extremidades da célula de Hadley, em direção aos polos, porque não foi comprovada essa evidencia, já que a redução máxima de precipitação foi observada em $40^{\circ} \mathrm{S}$, não coincidindo o ramo descendente da célula de Hadley para o continente Sul Americano. 
O deslocamento mais ao Sul da célula de Hadley pode estar associado aos eventos El Niño e Oscilação Sul (ENOS), o qual revelou um agrupamento estatisticamente significativo de sua circulação com El Niño. Para eventos neutros e La Niña, se observou pouca diferença na intensidade da célula no verão e inverno (STACHNIK e SCHUMACHER, 2011). Antes do período de 1980-2010, a existência de um padrão entre a célula de Hadley e os ENOS não foi observado. Isso pode sugerir que a associação da célula de Hadley com os ENOS apresenta uma forte influência da variabilidade multidecadal, pois antes e depois da mudança em meados da década de 1970, o clima apresentou respostas diferentes nesta região (QUAN et al., 2004).

Essa redução no vapor de água atmosférico para o Sul da América do Sul pode ser comprometedora para o regime hidrológico da região. A disponibilidade hídrica em países como a Argentina é, quase que na sua totalidade, suprida pelo desgelo que ocorre nos Andes. Isso pode ser agravante tendo em vista que a precipitação anual é inferior a $200 \mathrm{~mm} /$ ano para algumas regiões do Sul do continente (TRENBERTH, 1991).

A diminuição de vapor de água na atmosfera, além de contribuir para a redução da precipitação é um fator preponderante para a formação de neva nas montanhas andinas. Essa redução de vapor de água na troposfera pode comprometer o abastecimento de água para os países Sul Americano, como também comprometer sua agricultura. Essa redução no vapor de água para essas regiões pode trazer implicações para a precipitação, uma vez que em média, cerca de 70\% da precipitação é oriunda da umidade já existente na atmosfera no momento da formação da tempestade, enquanto o restante vem da evaporação, evapotranspiração ou da evolução da tempestade (TRENBERTH et al., 2007).

O transporte de umidade no Sul da Américo do Sul é praticamente de oeste ao longo de todo o ano, transportando umidade do Pacífico Sul para o Sul da Argentina e Chile. Durante os meses de inverno, com deslocamento do anticiclone do Pacífico ocorre o desenvolvimento de uma crista no campo de pressão sobre o continente. Este deslocamento faz com que seja produzindo uma componente zonal do gradiente de pressão para o lado ocidental do continente. Essa configuração contribui para o fluxo de vapor de água atinja os Andes, fazendo com que o fluxo de oeste se estende mais para o Norte (RAO et al., 2002).

Esta configuração faz com que durante os meses de agosto a novembro na estação 85799 , localizada no Chile, seja observada tendência positiva para o vapor de água atmosférico, enquanto que nas estações argentinas 87344 e 87576, sejam observadas tendências negativas. Devido ao encontro do fluxo de umidade com os Andes, a região a sotavento dos Andes até a Patagônia apresenta uma precipitação anual inferior a $200 \mathrm{~mm}$ /ano (TRENBERTH, 1991). Devido ao déficit de umidade na atmosfera, foram observadas as tendências negativas sobre o Sul da América do Sul. 
Projeções futuras realizadas por Levang e Schmitt (2015) mostraram que os giros oceânicos subtropicais podem diminuição na precipitação de acordo com modelos climáticos.

Observações realizadas por Vuille et al. (2008) comprovaram que a extensão das geleiras nos Andes encolheu. Em paralelo com o recuo das geleiras, o clima nos Andes tropical mudou significativamente ao longo dos últimos 60 anos, apresentando um aumento na temperatura de aproximadamente $0,1{ }^{\circ} \mathrm{C}$ por década, com diminuição da precipitação. Essa mudança observada tem potencial para afeta o balanço energético das geleiras por meio de sua sensibilidade a mudança na umidade atmosférica, precipitação, albedo e balanço de energia.

As simulações realizadas pelo IPCC (2013) indicam que as geleiras continuarão a recuar, com base em diferentes cenários para 2050 e 2080. Para algumas dessas geleiras as previsões é de que elas desapareçam em poucas décadas. Para as que não desaparecem completamente. Esperasse também mudança na sazonalidade das vazões devido à redução do gelo durante a estação seca, que irá afetar significativamente a disponibilidade de água a jusante. A redução do vapor de água na atmosfera pode ser um indicativo destas mudanças.

\section{CONCLUSÕES}

Identificou-se dois padrão distintos de tendência na concentração de vapor de água na baixa troposfera da América do Sul. O primeiro deste padrão, o de tendência positiva foi verificado para as estações localizadas nas regiões Norte, Central e Leste do continente sul americano. As tendências positivas foram observadas em quase todos os meses do ano nas estações que demonstraram aumento de vapor de água anual, sendo observada tendência estatisticamente significativa principalmente para os meses de primavera e outono.

O outro padrão observado foi o de tendência negativa, indicando redução na concentração de vapor de água. Essa tendência foi verificada na região Sul da América do Sul, compreendida pelo Chile a Argentina. As tendências mensais estatisticamente significativas demonstradas nestas estações foram predominantemente de redução, sendo observada nos meses de inverno, primavera e verão.

\section{REFERÊNCIAS}

ANDREOLI, R. V.; SOUZA, R. A. F.; KAYANO, M. T.; CANDIDO. L. A. Seasonal anomalous rainfall in the central and eastern Amazon and associated anomalous oceanic and atmospheric patterns. International Journal of Climatology, v. 32, n. 8, p. 1193-1205, 2012.। 
ALLAN, R. P. The Role of Water Vapour in Earth's Energy Flows. Surveys in Geophysics, v. 33, p. 557-564, 2012.

BARKHORDARIAN, A.; SAATCHI, S. S.; BEHRANGI, A.; LOIKITH, P. C.; MECHOSO, C. R. A Recent Systematic Increase in Vapor Pressure Defcit over Tropical South America. Scientific Reports, v. 9, p. 15331-15342, 2019.

BARKHORDARIAN, A.; VON STORCH, H., ZORITA, E.; LOIKITH, P. C.; MECHOSO, C. R. Observed warming over northern South America has an anthropogenic origin. Climate Dynamics, v. 51, n. 5, p. 1901-1914, 2017.

CAI, W.; LENGAIGNE, M.; BORLACE, S.; COLLINS, M.; COWAN, T.; MCPHADEN, M. J.; TIMMERMANN, A.; POWER, S.; BROWN, J.; MENKES, C.; NGARI, A.; VINCENT, A. More extreme swings of the South Pacific convergence zone due to greenhouse warming. Nature, v. 488, p. 365-369, 2012.

CHUNG, E. S.; SODEN, B.; SOHN, B. J.; SHI, L. Upper-tropospheric moistening in response to anthropogenic warming. Proceeding of the national Academy of Sciences of the United States of America, v. 111, n. 32, p. 11636-11641, 2014.

COHEUR, P. F. C.; CLERBAUX, C.; CARLEER, M.; FALLY, S.; HURTMANS, D.; COLIN, R.; HERMANS, C.; VANDAELE, A. C.; BARRET, B.; MAZIĖRE, M.; BACKER, H. Retrieval of atmospheric water vapor columns from FT visible solar absorption spectra and evaluation of spectroscopic databases. Journal of Quantitative Spectroscopy \& Radiative Transfer, v. 82, p.133-150, 2003.

CORRÊA, C. S.; WIEGAND, F.; DALlAROSA, R.; SENNA, R. C. Análise da ocorrência dos fluxos e jatos de nível baixo no perfil vertical do vento na baixa atmosfera em Manaus (AM). Revista Brasileira de Meteorologia, São Paulo, v. 23, p. 327-333, 2008.

DESSLER, A. E.; WONG, S. Estimates of the Water Vapor Climate Feedback during El NiñoSouthern Oscillation. Journal of Climate, v. 22, p. 6404-6412, 2009.

ENGLAND, M. H.; MCGREGOR, S.; SPENCE, P.; MEEHL, G. A.; TIMMERMANN, A.; CAI, W.; GUPTA, A. S.; MCPHADEN, M. J.; PURICH, A.; SANTOSO, A. Recent intensification of wind-driven circulation in the Pacific and the ongoing warming hiatus. Nature Climate Change, v. 4, p. 222-227, 2014.

FAO - Organización de las Naciones Unidas para la Alimentación y la Agricultura. Oficina Regional de la FAO para América Latina y el Caribe. FAO, 2019.

FISCH, G.; VENDRAME, I. F.; HANAOKA, P. C. M. Variabilidade espacial da chuva durante o experimento LBA/TRMM 1999 na Amazônia. Acta Amazônica, Manaus, v. 37, n. 4, p. 583-590, 2007.

FORSTER, P. M. F.; COLLINS, M. Quantifying the water vapour feedback associated with postPinatubo global cooling. Climate Dynamics, v. 23, p. 207-214. 2004.

HELD. I. M.; SODEN, B. J. Robust Responses of the Hydrological Cycle to Global Warming. Journal of Climate, v. 19, p. 23-35, 2006. 
HOUGHTON, J. T.; Y. DING, D. J.; GRIGGS, M.; NOGUER, P. J.; VAN DER LINDEN, X.; DAI, K.; MASKELL, C. A.; JOHNSON, E. Climate Change 2001: The Scientific Basis. Cambridge: University Press, 2001. 881p.

IPCC: Climate Change 2013: The Physical Science Basis. Contribution of Working Group I to the Fifth Assessment Report of the Intergovernmental Panel on Climate Change. STOCKER, T. F.; QIN, D.; PLATTNER, G. K.; TIGNOR, M.; ALLEN, S. K.; BOSCHUNG, J.; NAULES, A.; XIA, Y.; BEX, V.; MIDGLEY, P. M. (Eds.). Cambridge: Cambridge University Press, 2013. $1535 \mathrm{p}$.

KENDALL, M. G. Rank correlation methods. 4 ed. London: Charles Griffin, 1975. 163p.

LABRAGA, J. C.; FRUMENTO, O.; LÓPEZ, M. The Atmospheric Water Vapor Cycle in South America and the Tropospheric Circulation. Journal of Climate, v. 13, p. 1899-1915, 2000.

LEVANG, S. J.; R. W. SCHMITT. Centennial Changes of the Global Water Cycle in CMIP5 Models 5 American. Meteorological Society, v, 28, p. 6489-6502, 2015.

MANN, H. B. Non-parametric test against trend. Econometrika, v.13, p. 245-259, 1945.

MARENGO, J. A.; NOBRE1, C. A.; TOMASEllA, J.; CARDOSO, M. F.; OYAMA, M. D. Hydro-climatic and ecological behaviour of the drought of Amazonia in 2005. Philosophical Transactions of the Royal Society B: Biological Sciences, v. 363, p. 1773-1778. 2008.

MERCADO, L.M.; BELLOUIN, N.; SITCH, S.; BOUCHER, O.; HUNTINGFORD, C.; WILD, M.; COX, P. M. Impact of changes in diffuse radiation on the global land carbon sink. Nature, v. 458, p. 1014-1018, 2009.

MINSCHWANER, K.; DESSLER, A. E. Water Vapor Feedback in the Tropical Upper Troposphere: Model Results and Observations. Journal of Climate, v. 17, p. 1272-1282, 2004.

MINSCHWANER, K.; DESSLER. A. E.; AENGPHOKHAI, P. S. Multimodel Analysis of the Water Vapor Feedback in the Tropical Upper Troposphere. Journal of Climate, v. 19, p. 54555464, 2006.

PALTRIDGE, G.; ARKING, A.; POOK, M. Trends in middle- and upper-level tropospheric humidity from NCEP reanalysis data. Theoretical and Applied Climatology, v. 98, p. 351-359, 2009.

QUAN, X. W.; DIAZ, H. F.; HOERLING, M. P. Change in the tropical Hadley cell since 1950. In: DIAZ, H. F.; BRADLEY, R. S. (Org.) The Hadley Circulation: Past, Present and Future. New York: Cambridge, 2004. 120p.

RAO, V. B.; CHAPA, S. R.; FERNANDEZ, J. P. R.; FRANCHITO, S. H. A Diagnosis of Rainfall over South America during the 1997/98 El Ninõ Event. Part II: Roles of Water Vapor Transport and Stationary Waves. Journal of Climate, v. 15, p. 512-521, 2002.

RANDALL, D. A. Climate models and their evaluation. In: SOLOMON, S. Climate Change 2007: The Physical Science Basis. Contributions of Worf Group I to the Fourth Assessment Reporto f the Intergovernmental Panel on Climate Change. 2007, p. 591-662. 
SHINDELL, D. T. G. A.; SCHMIDT, R. L.; MILLER, D. R. Northern Hemisphere winter climate response to greenhouse gas, ozone, solar, and volcanic forcing. Journol of Geophysical Research Atmospheres, v. 106, p. 7193-7210, 2001.

SITCH, S.; COX, P.M.; COLLINS, W.J.; HUNTINGFORD, C. Indirect radiative forcing of climate change through ozone effects on the land-carbon sink. Nature, v. 448, p. 791-794, 2007.

SODEN, B. J.; HELD, I. M.; COLMAN, R. Quantifying Climate Feedbacks Using Radiative Kernels. Journal of Climate, v. 21, p. 3504-3520, 2008.

SOlOMON, S.; ROSENLOF, K. H.; PORTMANN, R. W.; DANIEL, J. S.; DAVIS, S. M.; SANFORD, T. J.; PLATTNER, G. K. Contributions of Stratospheric Water Vapor to Decadal Changes in the Rate of Global Warming. Science, v. 327, p. 1219-1223, 2010.

STACHNIK, J. P.; SCHUMACHER, C. A comparison of the Hadley circulation in modern reanalyses. Journal of Geophysical Research. v. 116, p. 234-246. 2011.

TAN, J.; JAKOB, C.; ROSSOW, W. B.; TSELIOUDIS, G. Increases in tropical rainfall driven by changes in frequency of organized deep convection. Nature, v. 519, p. 451-454, 2015.

TRENBERTH, K. E. Storm tracks in the Southern Hemisphere. Journal of Climate. v. 48, p. 21592178. 1991.

TRENBERTH, K. E.; DAVIS, C. A.; FASULLO, J. Water and energy budgets of hurricanes: Case studies of Ivan and Katrina. Journol of Geophysical Research Atmospheres. v. 112, p. 1-11, 2007.

VEIGA, J. A. P.; MARENGO, J. A.; RAO, V. R. A influência das anomalias de tsm dos oceanos atlântico e Pacífico sobre as chuvas de monção da América do Sul. Revista Brasileira de Meteorologia, São Paulo, v. 17, p. 181-194, 2002.

VERA, C.; BAEZ, J.; DOUGLAS, M.; EMMANUEL, C. B.; MARENGO, J.; MEITIN, J.; NICOLINI, M.; NOGUES-PAEGLE, J.; PAEGLE, J.; PENALBA, O.; SALIO, P.; SAUlO, C.; SILVA DIAS, M. A.; SILVA DIAS, P.; ZIPSER, E. The south american low-level jet experimente. Bulletin of the American Meteorological Society, v. 87, p. 63-77, 2006.

VUILLE, M.; FRANCOU. B.; WAGNON, P.; JUEN, I.; KASER, G.; MARK, B. G.; BRADLEY, R. S. Climate change and tropical Andean glaciers: Past, present and future. Earth Science Reviews, v. 89, p. 79-96. 2008.

WANDERLEY, H. S.; JUSTINO, F. B. Aumento da concentração de vapor de água atmosférico na Groenlândia. Ciência e Natura, Santa Maria, v. 37, p. 153-158, 2015.

WULFMEYER, V.; BAUER, H. S.; GRZESCHIK, M.; BEHRENDT, A.; VANDENBERGHE, F.; BROWELL, E. V. 4-dimensional variational assimilation of watervapor differential absorption lidar data: the first case study within IHOP_2002. Monthly Weather Review, v. 134, p. 209-230, 2006.

Trabalho enviado em $22 / 01 / 2020$

Trabalho aceito em 04/04/20 